\title{
The relevance internet users assign to algorithmic-selection applications in everyday life
}

\author{
Michael V. Reiss*, Noemi Festic, Michael Latzer, \& Tanja Rüedy, \\ University of Zurich, Department of Communication and Media Research IKMZ, Switzerland \\ *Corresponding author: m.reiss@ikmz.uzh.ch
}

\begin{abstract}
The rapidly growing academic and public attention to algorithmic-selection applications such as search engines and social media is indicative of their alleged great social relevance and impact on daily life in digital societies. To substantiate these claims, this paper investigates the hitherto little explored subjective relevance that Internet users assign to algorithmic-selection applications in everyday life. A representative online survey of Internet users comparatively reveals the relevance that users ascribe to algorithmic-selection applications and to their online and offline alternatives in five selected life domains: political and social orientation, entertainment, commercial transactions, socializing and health. The results show that people assign a relatively low relevance to algorithmic-selection applications compared to offline alternatives across the five life domains. The findings vary greatly by age and education. Altogether, such outcomes complement and qualify assessments of the social impact of algorithms that are primarily and often solely based on usage data and theoretical considerations.
\end{abstract}

\section{Keywords}

algorithmic governance, algorithmic selection, algorithms, subjective relevance, everyday life, survey data, social media

\section{Introduction}

Algorithmic selection is the automated assignment of relevance to certain selected pieces of information (Latzer, Hollnbuchner, Just, \& Saurwein, 2016). On social media, for instance, algorithmic selection is responsible to filter users' news feeds, to allocate advertisements, and to recommend specific content to users. The great attention toward algorithmic selection in public and academic debates reflects widespread assumptions that it has an extensive influence on daily life in digital societies (Beer, 2017; Gillespie, 2014; Latzer \& Just, 2020; Willson, 2017).

Such appraisals of the relevance of algorithmic selection, combined with assumptions on the associated risks including political and economic manipulation, discrimination, data breaches, and a biased perception of the world (Latzer et al., 2016), form the rationale for the need and kind of governance of algorithmic se- lection. Appropriate governance choice, however, calls for an accurate and up-todate understanding of the social relevance of algorithmic selection in order to, among other things, assess the scope and magnitude of potential risks associated with it. This paper aims to contribute to the systematic assessment of the social relevance of algorithmic selection in order to provide for a profound basis for governance measures. For this purpose, it suggests including the Internet users' assigned relevance of algorithmic-selection applications in such assessments.

Investigations about algorithmic selection all share the commonality that algorithms are a complex concept and difficult to grasp in empirical social science research (Kitchin, 2017). In practice, algorithmic selection is embedded in and applied by a wide and fast-growing range of online applications such as social media, search engines, news websites, or online shops. These applications are the place 
where users experience and are potentially influenced by algorithmic selection. Hence, the social relevance of algorithmic selection mainly unfolds via such applications and along the lines of users' usual but manifold and deliberate daily online practices (Bucher, 2017; Willson, 2017).

Previous studies have predominantly deduced the relevance of algorithmic selection and algorithmic-selection applications either from purely theoretical reasoning or from non-generalizable empirical investigations (Abril, 2016; Baek \& Kim, 2016; Beer, 2017; Park, 2019, Yang \& Men, 2020). These empirical accounts approximate the social relevance of algorithmic selection from a user perspective by measuring the amount and frequency of the use of algorithmic-selection applications and the effects of or attitudes toward them.

In order to question and substantiate these existing assessments and to gain a more holistic understanding of the relevance of algorithmic selection for Internet users' everyday life, this paper argues for an additional empirical indicator: the relevance that people subjectively assign to algorithmic-selection applications.

This approach takes into account that algorithmic selection is experienced by users often unknowingly in everyday situations: Although search engines fundamentally build on algorithmic selection, users may not be aware of it, for example. However, the social relevance of algorithmic selection, its benefits, and risks are provided with or without users' awareness. Asking users about concrete applications (e.g., Google Search) and not about the software technology that lies behind them is therefore imperative when aiming to assess the relevance of technologies like algorithmic selection in daily life that users might not even be aware of. The measurement of users' assigned relevance of algorithmicselection applications is hence a valuable, but hitherto missing piece in current efforts to assess the actual relevance of algorithmic selection in digital societies.

This paper uses the term algorithmic selection and not algorithm in order to highlight that the focus is on the so- cio-technical context algorithms are embedded in, and not merely on algorithms as technical artefacts (Latzer \& Festic, 2019). Consequently, this article chooses algorithmic-selection applications as its unit of analysis, as the tangible and accessible manifestation of algorithmic selection. Measuring the subjective relevance makes it possible to weight and better interpret existing findings on the overall social relevance of algorithmic selection that are solely based on the amount and frequency of use (Latzer \& Festic, 2019).

Drawing on a combination of qualitative interviews and a nation-wide, representative online survey of Swiss Internet users, this study examines five domains of everyday life: political and social orientation, entertainment, commercial transactions, socializing, and health. Furthermore, in order to establish a benchmark for the assessment of algorithmic-selection applications, the relevance of alternatives, i.e., non-algorithmic online and offline daily services and activities, such as reading news, watching television, and talking to friends, is investigated as well.

The main contributions of this article to the literature on the social relevance of algorithmic selection are its subjective user perspective, the comprehensive, empirical assessment of the assigned relevance of algorithmic-selection applications relative to online and offline alternatives, and comparisons between different life domains and socio-demographic groups. These representative results complement the current debate, promote more nuanced assessments, and may form the basis for empirically better-informed policy-making regarding the governance of algorithmic selection and algorithmic-selection applications. Such up-to-date, empirical results are especially essential in the light of ongoing discussions about regulatory interventions regarding social media, for example in the context of manipulation and biased political information (Bayer et al., 2019; European Commission, 2018).

The paper continues by providing a literature review on existing research regarding the social relevance of algorithmic selection in five life domains. Subsequent- 
ly, an overview of current measurements on the social relevance of algorithmic selection discusses suitable methodological approaches. Finally, the guiding research questions are derived, and the empirical research design is presented. The final sections summarize the results, discuss implications, and draw conclusions.

\section{The relevance of algorithmic selection - existing evidence for five life domains}

According to recent research, algorithmic selection is increasingly prevalent in peoples' everyday lives. As a result, algorithmic selection increasingly governs what Internet users see and, consequently, how people perceive the world (Just \& Latzer, 2017). The distinction between the following five life domains observed in this paper: political and social orientation, entertainment, commercial transactions, socializing, and health helps to investigate ramifications of algorithmic selection in a more nuanced way:

The life domain social and political orientation has so far received the most attention from research on the social relevance of algorithmic selection. The widest academic focus lies on the usage time of online services for political topics (Baek \& Kim, 2016; Gil de Zúñiga, Ardèvol-Abreu, \& Casero-Ripollés, 2021; Karakaya \& Glazier, 2019; Lee, Lee, So, Leung, \& Chan, 2017; Park, 2019; Vraga \& Tully, 2021; Westerwick, Johnson, \& Knobloch-Westerwick, 2017; Yang \& Men, 2020). The results indicate increased social media use for information seeking (Newman, Fletcher, Schulz, And1, \& Nielsen, 2020; Shearer, 2018) and the consideration of online services as alternative daily news sources (Althaus \& Tewksbury, 2000; Bialik \& Matsa, 2017; Schmidt, Merten, Hasebrink, Petrich, \& Rolfs, 2019). Facebook's news feed algorithm's logic can also directly influence news production and lead to increasingly similar content across different media outlets (Caplan \& Boyd, 2018).

Mainly through the emergence of online applications like Spotify, YouTube, or
Netflix, which automatically recommend content to individual users, algorithmic selection has also become key for everyday entertainment. However, more traditional recommendations have repeatedly been shown to influence everyday music consumption more heavily (Hamilton, 2019), although there are different usage types for which applications based on algorithmic selection are not equally relevant (Lepa \& Hoklas, 2015).

Algorithmic selection increasingly accompanies people's daily commercial transactions, including recommender systems and the allocation of personalized advertisements. While the advertising industry heavily relies on algorithmic allocation of user-specific content (eMarketer, 2020), various findings from a user perspective show that users mainly perceive algorithmically allocated advertisements as useless, inaccurate, or even offensive (De Keyzer, Dens, \& De Pelsmacker, 2015; Kim \& Huh, 2017; Smit, Van Noort, \& Voorveld, 2014). This rather negative attitude likely reflects concerns caused by the collection of user data (Phelan, Lampe, \& Resnick, 2016). Furthermore, with regard to product recommendations, scientific findings show that even though algorithmic recommender systems may be considered helpful (Chen, 2012), they lead to less conversion than recommendations from real people, such as other Internet users (Lin, 2014).

With regard to socializing, algorithmic selection increasingly governs the interaction between Internet users (Bucher, 2012, 2017; Celik \& Dokuz, 2018). For instance, by rating and scoring user profiles, algorithmic selection is responsible deciding who is considered a potential friend on social network sites or a match on dating services. In terms of dating services, recent studies show that these services are especially of interest for people belonging to societal minorities, such as the LGBT community (Sumter \& Vandenbosch, 2019; Wang, 2020). On the one hand, these applications likely facilitate the social interaction not only within but also across various societal groups. On the other hand, scholars have raised concerns that an in- 
creased governance of rating and scoring algorithms likely fuels existing discrimination and strengthens biases (Courtois \& Timmermans, 2018; Wang, 2020). However, to better assess who is most likely to be exposed to these risks, more research that takes account of different societal groups is needed.

People have increasingly been seeking health information online for a long time (Rains, 2007). More recently, self-tracking devices that gather vital data can be empowering for patients when dealing with medical professionals (Lomborg \& Frandsen, 2016). They are positively related to the overall health status and can be a superior alternative to traditional paper-and-pencil tracking (Abril, 2016). Their adoption depends on various characteristics of the devices (Adapa, Nah, Hall, Siau, \& Smith, 2018) as well as user and context variables (Canhoto \& Arp, 2017). Using self-tracking devices to monitor vital aspects about oneself can result in measurable, transparent, and connected bodies. This consequence has been called "algorithmic skin" (Williamson, 2015).

As this literature review shows, comprehensive research with respect to algorithmic selection is lacking, especially when aiming to compare the relevance of algorithmic-selection applications to alternatives, such as print media or human interactions apart from the digital sphere. Furthermore, to better assess risk exposure, there are no comparative findings on the social relevance of algorithmic selection that take different societal groups into account. Beyond this, as the following chapter shows, there is a methodological research gap regarding the relevance that people subjectively assign to algorithmic-selection applications.

\section{Approaches to measuring the social relevance of algorithmic selection: An overview}

The assessment of the social relevance of algorithmic selection and risks arising from its applications as well as related initiatives to regulate such services have predominantly been based on purely theoretical reasoning and their mere existence (Pariser, 2011; Seaver, 2019). However, there is an increasing number of empirical approaches illuminating this topic using different methodological designs to expand the understanding about algorithmic selection and its societal relevance (Kitchin, 2017), each with their own advantages and disadvantages. Subsequently, an overview of the methodological approaches to measuring the social relevance of algorithmic selection is given. From this review, we derive the need for including measures on the subjective significance assigned to algorithmic-selection applications and proceed to contribute to filling this gap.

Existing empirical research on the social relevance of algorithmic selection can be divided into two broad perspectives: a bottom-up user, or a top-down supplier perspective. Of the two, the former is by far the more popular and frequent approach. The user perspective is mainly acquired by collecting self-reported data in order to approximate individuals' Internet behavior (de Vreese \& Neijens, 2016), predominantly relying on surveys of the amount and frequency of usage of online services. Repertoire studies also fall into this category and they increasingly take online sources including social media into consideration, enabling a partial assessment of the social relevance of algorithmic-selection applications. To avoid potentially biased self-reported data, a rather novel strand of research - which, like the previously mentioned approaches, also utilizes the amount and frequency of usage of algorithmic-selection applications as a proxy for their relevance - gathers respective data by tracking online behavior (Kilger \& Romer, 2013; Mattlin \& Gagen, 2013). Studies based on tracking data are still quite rare and often limited to social media behavior (Deng et al., 2019; Junco, 2013).

Self-reported data and tracking data are also combined and compared in order to investigate usage time as a proxy for the social relevance of algorithmic-selection applications (Thorson, Cotter, Medeiros, \& Pak, 2021). Results reveal that self-report- 
ed data are often inaccurate because people are likely to overestimate the time they spend online (Araujo, Wonneberger, Neijens, \& de Vreese, 2017; Deng et al., 2019; Guess, Munger, Nagler, \& Tucker, 2019; Junco, 2013; Scharkow, 2016). This suggests that even though self-reported usage time is widely employed, it does not permit precise but rather distorted assessments of the relevance of algorithmic-selection applications. But tracking data can also be subject to specific biases, e.g., self-selection (Jürgens, Stark, \& Magin, 2019). Another limitation is its methodological restriction to the online sphere, hence being insufficient to appraise the social relevance of algorithmic-selection applications compared to offline alternatives.

A limited number of qualitative studies consider a broader range of settings where people rely on algorithmic selection in daily situations (Bucher, 2017; Festic, 2020) and allow a more in-depth understanding of their social relevance. Qualitative studies also rely on self-reporting from a user perspective. In contrast to quantitative survey data, they provide a more indepth understanding, for example, of the embeddedness of algorithmic-selection applications in Internet users' daily practices but lack generalizability across services and life domains.

In addition to studies on the usage of algorithmic-selection applications, attitudes toward them are examined to derive their social relevance from users' reliance on them, mainly measured through the credibility ascribed to algorithmically produced content. These studies can produce contradictory findings. On the one hand, research indicates that people may be rather skeptical toward applications that build on algorithmic selection (Logg, Minson, \& Moore, 2019; Promberger \& Baron, 2006), and on the other hand, Internet users are more likely to adhere to advice proposed by algorithms as opposed to human sources (Logg et al., 2019).

The social relevance of algorithmic selection is also assessed by directly investigating the effects that the use of algorithmic-selection applications has on individuals' attitudes and behaviors, instead of indirectly inferring them from theoretical reasoning or mere usage data. Effect studies usually apply experimental settings.

Lastly, there are endeavors to measure the social relevance of algorithmic selection taking the top-down, supplier-side perspective by simulating algorithms (Möller, Trilling, Helberger, \& van Es, 2018), reverse-engineering algorithmic program code (Diakopoulos, 2015), or interviewing programmers (Rosenberg, 2008) in order to understand exactly how algorithms seek to and may actually influence Internet users' everyday lives. Table 1 provides an overview of the existing methodological approaches presented in this chapter.

Table 1: Existing methodological approaches to measuring the social relevance of algorithmic selection

\begin{tabular}{|l|l|l|l|}
\hline Who? & What? & How? \\
\hline \multirow{4}{*}{$\begin{array}{l}\text { User } \\
\text { perspective } \\
\text { (bottom- up) }\end{array}$} & $\begin{array}{l}\text { Usage } \\
\text { (amount/ } \\
\text { frequency/ } \\
\text { repertoires) }\end{array}$ & Quantitative & Surveys \\
\cline { 2 - 3 } & Effects & Eualitative & Tracking \\
\cline { 2 - 3 } & & Interviews \\
\cline { 2 - 3 } & Attitudes & Surveys \\
\hline \multirow{2}{*}{$\begin{array}{l}\text { Supplier } \\
\text { perspective } \\
\text { (top-down) }\end{array}$} & Code & Reverse engineering \\
\cline { 2 - 3 } & Output & Simulations \\
\cline { 2 - 3 } & Input & Interviews programmers \\
\hline
\end{tabular}

We argue that one crucial missing piece in this field of research is to ask the users how relevant they regard algorithmic-selection applications to be for their lives. There have been limited endeavors to fill this gap for single issues such as gathering information on the 2016 US presidential campaign (Gottfried, Barthel, Shearer, \& Mitchell, 2016), but comprehensive empirical assessments are lacking.

The methodological approach used in this article fills this gap: We take a user perspective and aim at empirically approximating the social relevance of algorithmic selection by measuring the subjective relevance Internet users assign to algorithmic-selection applications. This approach 
will be introduced in greater detail subsequently.

\section{Introducing assigned relevance as a measurement of the social relevance of algorithmic selection}

To measure the social relevance of algorithmic selection, empirical approaches have already addressed a few important questions but also come with limitations, as outlined above. We propose exploring subjectively assigned relevance as a complementary measurement in quantitative surveys in order to provide more comprehensive, nuanced empirical assessments of the social relevance of algorithmic selection in people's daily lives.

Algorithmic selection is associated with a variety of social risks to which Internet users are often understood to be highly vulnerable and helplessly exposed. Such a view widely neglects Internet users' agency by underestimating their capacity to manage their Internet use and its consequences. It has been shown, for example, that people are well aware and make sense of the algorithms they encounter online (Bucher, 2017), apply various practices to deal with them (van der Nagel, 2018), and thereby significantly shape algorithms in turn. Hence, it seems vital to investigate individuals' perceptions of the relevance of algorithmic selection.

Previous studies have shown that perceptions of relevance and preferences are likely to differ from usage time and should therefore be considered an additional element in assessing the social relevance of algorithmic selection (Festic, 2020; Swart, Peters, \& Broersma, 2017). For example, people may use social media very extensively but still rate information from a printed newspaper as more relevant and more influential for their social and political orientation, even though they spend much less time on it. The primary purposes for which people use social media are not necessarily information seeking but rather being entertained, passing time, or maintaining social relations (QuanHaase \& Young, 2010; Whiting \& Williams,
2013). Consequently, empirical data on the relevance subjectively assigned to algorithmic-selection applications considering users' perceptions and preferences is required in order to interpret and weight data on the usage of these services. By functioning as an additional, weighting dimension, the subjective relevance complements existing findings, allows for a more differentiated interpretation of them, and contributes to a more nuanced assessment of the social relevance of algorithmic-selection applications. When people are asked to assess the relevance of a service or activity it is intentionally left to them to intuitively decide how they conceptualize relevance in the given context; for example, why they assess online games as very relevant for their daily entertainment. Although people might have varying concepts of relevance or reasons for their evaluation, this openness assures that the relevance is assessed exactly as each individual finds it most appropriate. This leads to the intended unbiased subjective relevance assessment. The reasons behind a certain subjective relevance assessment can be manifold but are not the focus of this study.

Another argument for the measurement of subjectively assigned relevance is that people's perceptions of the relevance of algorithmic-selection applications are likely to influence how concerned they are about potential risks. Regardless of whether these concerns are justified or not, they are likely to affect users' protective behavior (e.g., deleting cookies), which in turn affects their exposure to risks of algorithmic-selection applications (e.g., biases by search engines or manipulations by targeted ads).

To conclude, investigating what people regard as relevant contributes another component to the empirical assessment and understanding of the social relevance of algorithmic selection.

Following the tenets of media repertoire research (Hasebrink \& Domeyer, 2012), the social relevance of algorithmic selection can only be accurately assessed when taking individuals' media repertoires into account as comprehensively 
as possible. For example, to measure the relevance of algorithmic-selection applications for people's everyday entertainment, it is imperative (1) to assess all the services and activities that individuals use for entertainment purposes in their everyday life and (2) to compare the relevance of algorithmic-selection applications with the relevance of their alternatives. Scholars agree that Internet users' news repertoire should be considered cross-media, since in a digitized environment recipients can choose between a growing number of media outlets (Dimmick, Chen, \& Li, 2004; Picone, Courtois, \& Paulussen, 2015; Schmidt et al., 2019; Swart et al., 2017). Accordingly, the following research question is at the core of this article (RQ1): What subjective relevance do Internet users assign to algorithmic-selection applications relative to online and offline alternatives?

A large amount of research theoretically discusses the social relevance of algorithmic selection in the context of everyday life (Bucher, 2017; Willson, 2017). Because of the great public interest in effects of algorithmic selection on news consumption, there has been a strong focus on this life domain. But algorithmic-selection applications are also important to several other domains of everyday life. More comparative research is needed to assess their social relevance across these domains because any governance of algorithmic selection ideally requires considering the manifold contexts in which algorithmic-selection applications operate and hence the varying social relevance thereof. This leads to the second research question (RQ2): How does the subjective relevance assigned to algorithmic-selection applications differ across five selected life domains (political and social orientation, entertainment, commercial transactions, socializing, and health)?

Lastly, it is likely that the subjective relevance of algorithmic-selection applications is not equally distributed within a society. Previous findings show, for example, that younger Internet users rely on certain algorithmic-selection applications more heavily than older Internet users, including social media (Shearer \& Matsa,
2018; Gottfried et al., 2016; Shearer, 2018), online dating (Smith, 2016; Sumter \& Vandenbosch, 2019), and mobile fitness tracking (Abril, 2016). However, so far, there are no findings to evaluate whether social groups with higher levels of usage time also assign higher levels of relevance to those algorithmic-selection applications. Hence, to better grasp whether certain social groups are more exposed to risks associated with algorithmic selection, information on subjective relevance is needed as an additional dimension to better interpret existing findings on frequency and amount of use. This is why the third research question addresses these differences (RQ3): How are socio-demographic variables (gender, age, education, income, and region) and personal characteristics (political interest, Internet use) associated with the subjective relevance that individuals assign to algorithmic-selection applications?

In order to answer these research questions, this article relies on a combination of qualitative interviews and quantitative survey data.

\section{Measuring subjectively assigned relevance}

This study consists of a mix of a qualitative (1) and a quantitative (2) phase, which are both described in-depth below.

\subsection{Data collection}

(1) Between June and August 2018, qualitative interviews were conducted with Swiss Internet users on the relevance of algorithmic-selection applications, the awareness of risks associated with algorithmic selection, and related questions (Festic, 2020). The interviewees were recruited through leaflets that were spread as widely as possible (train stations, fitness centers, youth clubs, retirement homes, restaurants, etc.) and received a gift card as a remuneration for their participation. The face-to-face interviews were conducted in German by a team of three researchers and lasted one hour on average. To ensure 
a congruent planning, data collection, and interpretation, all team members collaborated closely during all phases of the interviewing process.

(2) The quantitative survey data were collected between November 2018 and January 2019. Participants were recruited from an existing Internet panel by an independent market research company and received a small pecuniary incentive for their participation. The samples for the online survey and the qualitative interviews did not overlap. All participants in the quantitative survey gave informed consent about their participation and the research design was approved by the university's ethics review board. The survey lasted 30 minutes on average and covered topics such as attitudes towards algorithmic selection, risk assessments, awareness of algorithmic selection, and the subjective relevance assigned to algorithmic-selection applications and online and offline alternatives.

As stated above, both the interviews and the survey relied on five life domains. The classification of life domains was adopted from Büchi, Just, \& Latzer's (2016) analysis of the most widespread Internet activities in Switzerland.

\subsection{Sample characteristics}

Both the qualitative and the quantitative empirical parts of the study relied on a sample of Swiss Internet users. In Switzerland, $92 \%$ of the population used the Internet in 2019. Hence, Switzerland continually ranks among the highest-diffu- sion countries worldwide, similar to other Western countries (Latzer, Büchi, \& Festic, 2020).

(1) The sample for the qualitative interviews consisted of 58 Swiss Internet users and was composed applying a conscious choice and with the goal of reaching maximum variation within the sample regarding age, gender, education, and amount of Internet use (Festic, 2020).

(2) The sample for the quantitative survey comprised 1202 participants and is representative of the Swiss online population over the age of 16 with respect to age, gender, language region, household size, and employment status. Table 2 describes the sample characteristics in detail.

\subsection{Measures}

(1) In the qualitative interviews, we asked the interviewees to name algorithmic-selection applications, online non-algorithmic selection, and offline services and activities that are relevant for the life domains under investigation. Applying a sorting technique (Hasebrink \& Hepp, 2017), the interviewees named and ranked the activities and services they mentioned. For example, being on social media (algorithmic-selection application), calling on Skype (non-algorithmic selection online service), or meeting friends (offline activity) are among the relevant services and activities for the life domain of socializing. Interviewees sometimes had varying conceptualizations of how they define relevance but all could easily solve the task and give reasons for their choices.

Table 2: Sample characteristics

\begin{tabular}{llr}
\hline & Mean (SD) & Percentage (N) \\
\hline Age & $43.5(15.91)$ & $49 \%(590)$ \\
Female & & $66 \%(797)$ \\
Secondary education & & $25 \%(301)$ \\
Higher education & $6001-8000$ & \\
Income (CHF per month, median category) & $3.33(1.35)$ & $72 \%(865)$ \\
Political interest (5-point likert scale, 5 = high interest) & $3.52(2.82)$ & $24 \%(288)$ \\
Internet use (hours per day) & & $4 \%(49)$ \\
German-speaking & & \\
French-speaking & & \\
Italian-speaking & & \\
\hline
\end{tabular}


(2) The aggregated list was used as a basis for the development of the questionnaire for the subsequent quantitative online survey. The survey participants were asked to assess the relevance of the list of given services and activities for five life domains on a 5-point Likert scale with $1=$ "not at all relevant" and $5=$ "very relevant". For each of the five life domains, participants had to assess ten to fourteen services and activities, comprising algorithmic-selection applications, as well as non-algorithmic selection online and offline services and activities. In order not to restrict the subjectivity of participants, the survey questions on the relevance assessment were intentionally left open and non-leading to reflect and allow for varying concepts of relevance participants might have. Non-users of "social media" and "YouTube etc." did not have to state their relevance for the respective service and were hence assigned the lowest relevance score "not at all relevant".

It is important to note that for both the qualitative interviews and the quantitative survey, the participants were asked to rate the relevance they assigned to a list of different services and activities for different life domains. They were not given any information about whether the services and activities under investigation were based on algorithmic selection or not. Rather, the team of researchers classified the services and activities according to Latzer et al.'s (2016) definition of algorithmic selection. This approach appears appropriate given the black-box nature of algorithms and the oftentimes low awareness of algorithmic selection among Internet users. Furthermore, where possible, participants were not asked for specific services or activities but for the broader category of similar services or activities (e.g., "music streaming services such as Spotify, Soundcloud, iTunes").

\subsection{Analysis}

(1) The qualitative interviews were audiotaped and transcribed verbatim. Using the qualitative data analysis software MAXQDA, we composed a list of mentioned services and activities for all life domains which served as an input for the development of the questionnaire. This approach appeared fruitful since the subjectively assigned relevance to algorithmic-selection applications has not been empirically addressed hitherto and sufficient literature for the development of the survey questions and items was lacking.

(2) The dependent variable of interest in the quantitative data is the relevance participants assigned to various services and activities. To answer the first and second research question, the distribution of the ascription of relevance and means for all services and activities grouped by life domains are presented. This provides a comprehensive overview of the relevance assigned to algorithmic-selection applications and to their online and offline alternatives. Moreover, similarities, differences, and general patterns regarding the assignment of relevance to algorithmic-selection applications in five life domains are identified. The third research question is approached by exploring the influence of socio-demographic characteristics on the individual assignment of relevance. Standardized linear regression models for selected activities and services show its association with age, gender, education, income, political interest, Internet use, and language region.

\section{Results}

Figures 1 and 2 present the distribution of the subjectively assigned relevance (lower $\mathrm{x}$-axis) to respective activities and services in five life domains as well as the mean relevance attribution (vertical bars, higher $\mathrm{x}$-axis) by life domain. The activities and services are sorted in descending order regarding the mean relevance assignment. Algorithmic-selection applications are in bold while online alternatives are in italics.

Results for the political and social orientation life domain can be interpreted as follows. Participants assigned the relevance that 13 activities and services had for their individual orientation on political and societal issues. "Offline contacts" such 
as talking to family and friends were not only most frequently assigned the highest relevance score (45\%) but also had the highest mean relevance. With the "voting booklet" (a printed information brochure that is mailed to every Swiss household prior to each vote), "traditional TV/radio", and "print media" ranking second to fourth, offline alternatives were assigned the highest relevance. "Social media", an algorithmic-selection application, was ascribed the lowest relevance of all activities, both when looking at the frequency of the highest relevance score $(3 \%)$ and measured by the mean (2.10). Ranking fifth (3.28), "online news media" was attributed the highest relevance of all algorithmic-selection applications for political and social orientation, closely followed by "Wikipedia" and "search engines".

The results for the other four life domains are presented in figure 2 in analogue form. In the commercial transactions life domain, "online reviews" and "online shops" were amongst the most relevant services and activities. They seem to have substituted traditional alternatives substantially and were more relevant than other algorithmic-selection applications such as "personalized ads". In the health domain, algorithmic-selection applications ("health websites”, "Wikipedia”, "search en- gines") were reported as relevant, though still less relevant than "offline contacts" or "blood pressure etc.". The results further suggest that people rather rely on non-algorithmic activities to keep in touch and to meet new people (socializing).

Uniformly across all domains, offline alternatives ranked comparatively high whereas algorithmic-selection applications, in particular "social media", were assigned a low relevance. This was especially the case in the entertainment and socializing domains.

Table 3 on the page after next summarizes the results of five standardized linear regression models on the relevance of "social media" for the five life domains, controlling for the participants' socio-demographics. Coherently across all five life domains, increasing age was associated with a lower assignment of relevance for "social media". Except for the socializing domain, the same applied for having higher education, whereas increased Internet use was associated with higher assignments of relevance to "social media" for all life domains. For all domains, the effects of education were the greatest, followed by age. No uniform pattern was found for gender, income, political interest, and different regions.

Figure 1: Subjectively assigned relevance for political and social orientation

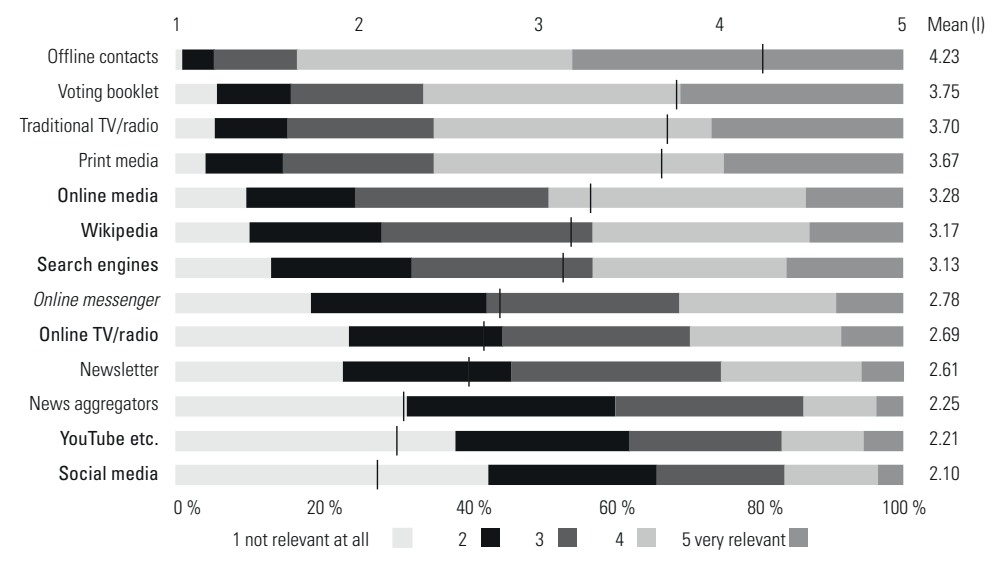

Note: The question was formulated as follows: Please assess the relevance of the following services and activities for your orientation on political and societal issues. $\mathrm{N}=1,202$. Algorithmic-selection applications are in bold, other online services in italics. 

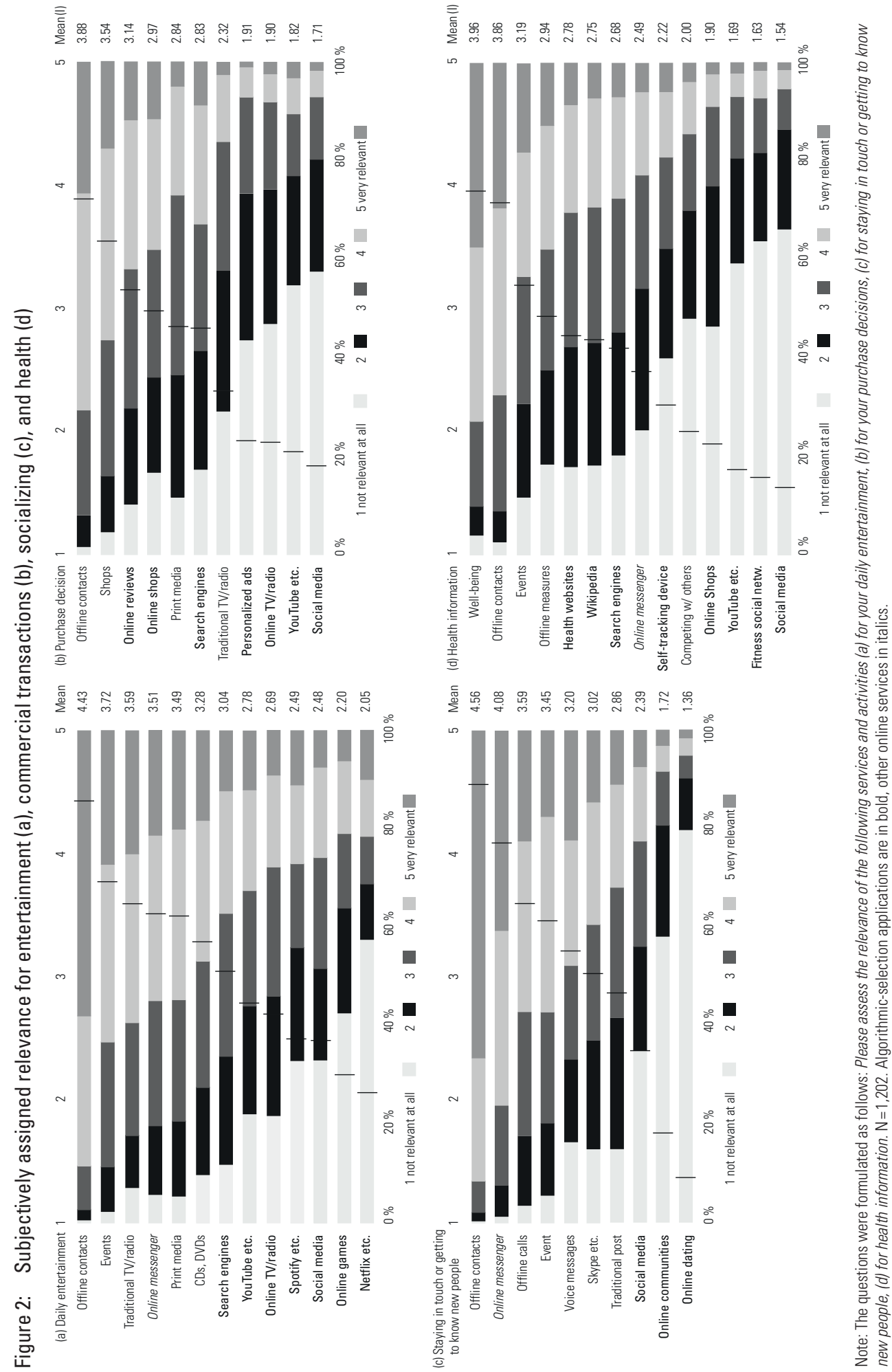
Table 3: Assigned relevance to social media in five life domains

\begin{tabular}{|c|c|c|c|c|c|c|c|c|c|c|}
\hline \multirow[b]{2}{*}{ Age } & \multicolumn{2}{|c|}{ Pol./soc. orientation } & \multicolumn{2}{|c|}{ Entertainment } & \multicolumn{2}{|c|}{ Commercial trans. } & \multicolumn{2}{|c|}{ Socializing } & \multicolumn{2}{|c|}{ Health } \\
\hline & -.216 & $(.034)^{* * *}$ & -.294 & $(.033)^{* * *}$ & -.234 & $(.034)^{* * *}$ & -.254 & $(.033)^{* * *}$ & -.124 & $(.035)^{* * *}$ \\
\hline Female & .038 & $(.032)$ & .071 & $(.031)^{*}$ & .030 & $(.034)$ & .052 & $(.032)$ & .013 & $(.034)$ \\
\hline Sec. education & -.272 & $(.153)$ & -.262 & (.148) & -.492 & $(.186)^{* *}$ & -.188 & (.148) & -.388 & $(.189)^{*}$ \\
\hline High. education & -.508 & $(.159)^{* *}$ & -.435 & $(.155)^{* *}$ & -.614 & $(.194)^{* *}$ & -.291 & (.156) & -.497 & $(.194)^{*}$ \\
\hline Income & -.042 & (.032) & -.013 & $(.032)$ & -.021 & $(.034)$ & .002 & $(.032)$ & -.074 & $(.034)^{*}$ \\
\hline Political interest & -.065 & (.035) & -.056 & $(.034)$ & -.106 & $(.036)^{* *}$ & -.044 & $(.034)$ & -.057 & $(.036)$ \\
\hline Internet use & .104 & $(.034)^{* *}$ & .113 & $(.03)^{* * *}$ & .104 & $(.034)^{* *}$ & .115 & $(.033)^{* * *}$ & .098 & $(.040)^{*}$ \\
\hline French-speaking & .134 & $(.076)$ & .122 & $(.074)$ & .033 & $(.080)$ & .221 & $(.075)^{* *}$ & .166 & $\left(.080^{*}\right)$ \\
\hline Italian-speaking & -.049 & (.151) & -.055 & $(.129)$ & -.100 & (.113) & -.134 & $(.124)$ & .023 & $(.162)$ \\
\hline $\mathrm{R}^{2}$ & .122 & & .163 & & .140 & & .129 & & .075 & \\
\hline Adj. $R^{2}$ & .114 & & .156 & & .133 & & .121 & & .067 & \\
\hline Num. obs. & 1043 & & 1044 & & 1045 & & 1044 & & 1041 & \\
\hline RMSE & .926 & & .910 & & .933 & & .924 & & .969 & \\
\hline
\end{tabular}

Table 4 presents standardized linear regression models on the association of the assignment of relevance regarding selected services and activities with sociodemographic variables. These models represent typical patterns and noteworthy cases. An overview of all activities and services can be found in the online supplement to this study.

Altogether, a few patterns emerged across the five life domains for their associations with different socio-demographic variables. Age was positively associated with the relevance assigned to "print media", and, with only a few exceptions, age and the relevance assigned to algorithmic-selection applications were negatively associated.

Across all life domains, being female was associated with a greater relevance assigned to offline activities and a lower one to algorithmic-selection applications. However, there were noteworthy exceptions with contrary relationships: "social media" for entertainment (see table 3), "traditional TV/radio" for commercial transactions, and "health websites" and "competing with others" in the life domain health.

Higher education was negatively associated with some algorithmic-selection applications such as "social media" and "YouTube etc." for the life domains political and social orientation, entertainment, commercial transactions, and health.
Overall and for most services and activities, education had the greatest effect.

In contrast, a greater amount of Internet use was never negatively associated with the relevance assigned to any algorithmic-selection application. Often, there was a positive effect of a greater amount of Internet use on the assignment of the relevance of algorithmic-selection applications. The level of Internet use had no significant effect on most offline activities and services.

\section{Discussion}

This paper argues for the inclusion of the perspective of subjectively assigned relevance in order to adequately assess the relevance of algorithmic selection in Internet users' daily lives. Qualitative interviews and a representative survey were conducted in Switzerland to assess the relevance that people assign to various algorithmic-selection applications and to their online and offline alternatives in five life domains. The findings substantiate current claims regarding the social implications of algorithmic-selection applications and can contribute to an empirically better-informed basis for policy-making regarding the governance of algorithmic selection. Evaluating the usage time of a specific algorithmic-selection application 
Table 4: Assigned relevance for selected services and activities in five life domains

\begin{tabular}{|c|c|c|c|c|c|c|c|c|c|c|c|c|}
\hline \multirow[b]{3}{*}{ Age } & \multicolumn{6}{|c|}{ Political and social orientation } & \multicolumn{6}{|c|}{ Entertainment } \\
\hline & \multicolumn{2}{|c|}{ Search engines } & \multicolumn{2}{|c|}{ Print media } & \multicolumn{2}{|c|}{ Voting booklet } & \multicolumn{2}{|c|}{ Netflix etc. } & \multicolumn{2}{|c|}{ YouTube etc. } & \multicolumn{2}{|c|}{ Events } \\
\hline & .017 & (.036). & .203 & $(.031)^{* * *}$ & -.108 & $(.033)^{* *}$ & -.311 & $(.034)^{* * *}$ & -.226 & $(.034)^{* * *}$ & .018 & $(.035)$ \\
\hline Female & .015 & $(.034)$ & .091 & $(.031)^{* *}$ & .077 & $(.033)$ & -.039 & $(.031)$ & -.153 & $(.031)^{* * *}$ & .121 & $(.032)^{* * *}$ \\
\hline Sec. education & -.063 & $(.123)$ & -.120 & $(.155)$ & .117 & (.134) & -.385 & $(.146)^{* *}$ & -.350 & $(.142)^{*}$ & .315 & $(.151)^{*}$ \\
\hline High. education & -.222 & $(.135)$ & .057 & (.124) & .128 & $(.146)$ & -.423 & $(.154)^{* *}$ & -.348 & $(.149)^{*}$ & .531 & $(.160)^{* * * *}$ \\
\hline Income & .007 & $(.033)$ & .074 & $(.032)^{*}$ & .030 & $(.033)$ & .029 & (.033) & -.066 & $(.030)^{*}$ & -.046 & $(.032)$ \\
\hline Political interest & -.122 & $(.036)^{* * *}$ & .218 & $(.034)^{* * *}$ & .221 & $(.036)^{* * *}$ & -.058 & (.034) & -.048 & $(.034)$ & .123 & $(.035)^{* * *}$ \\
\hline Internet use & .074 & $(.036)^{*}$ & -.066 & (.035) & -.021 & (.039) & .141 & $(.035)^{* * *}$ & .148 & $(.033)^{* * * *}$ & -.041 & (.032) \\
\hline French-speaking & -.079 & $(.078)$ & -.119 & (.069) & .041 & (.075) & -.006 & $(.073)$ & .043 & $(.074)$ & -.044 & (.075) \\
\hline Italian-speaking & -.115 & $(.126)$ & .404 & $(.130)^{* *}$ & .436 & $(.130)^{* * *}$ & -.049 & (.112) & -.034 & (.123) & -.074 & $(.140)$ \\
\hline $\mathrm{R}^{2}$ & .028 & & .137 & & .052 & & .175 & & .140 & & .045 & \\
\hline Adj. $R^{2}$ & .019 & & .129 & & .044 & & .168 & & .132 & & .036 & \\
\hline Num. obs. & 1041 & & 1043 & & 1040 & & 1011 & & 1045 & & 1044 & \\
\hline RMSE & .985 & & .917 & & .964 & & .898 & & .921 & & .958 & \\
\hline
\end{tabular}

\begin{tabular}{|c|c|c|c|c|c|c|c|c|c|c|c|c|}
\hline \multirow[b]{3}{*}{ Age } & \multicolumn{4}{|c|}{ Commercial transactions } & \multicolumn{4}{|c|}{ Socializing } & \multicolumn{4}{|c|}{ Health } \\
\hline & \multicolumn{2}{|c|}{ Online shops } & \multicolumn{2}{|c|}{ Personaliz. ads } & \multicolumn{2}{|c|}{ Online dating } & \multicolumn{2}{|c|}{ Onl. messenger } & \multicolumn{2}{|c|}{ Wearables } & \multicolumn{2}{|c|}{ Well-being } \\
\hline & -.181 & $(.33)^{* * *}$ & -.101 & $(.036)^{* *}$ & -.127 & $(.036)^{* * *}$ & -.155 & $(.032)^{* * * *}$ & -.068 & (.035) & -.052 & (.033) \\
\hline Female & -.098 & $(.032)^{* *}$ & -.088 & $(.034)^{* *}$ & -.117 & $(.031)^{* * *}$ & .180 & $(.030)^{* * *}$ & .004 & $(.034)$ & .107 & $(.032)^{* * *}$ \\
\hline Sec. education & -.101 & $(.129)$ & -.330 & $(.172)$ & -.304 & $(.186)$ & .251 & $(.130)$ & .047 & $(.143)$ & .083 & $(.137)$ \\
\hline High. education & -.105 & $(.141)$ & -.430 & $(.181)^{*}$ & -.420 & $(.191)^{*}$ & .361 & $(.137)^{* *}$ & .052 & (.155) & .215 & (.148) \\
\hline Income & .021 & $(.031)$ & .012 & $(.034)$ & -.087 & $(.035)^{*}$ & -.002 & $(.031)$ & -.002 & $(.034)$ & -.021 & $(.031)$ \\
\hline Political interest & -.036 & $(.034)$ & -.434 & $(.034)$ & .018 & $(.034)$ & .076 & $(.032)^{*}$ & -.050 & $(.035)$ & .117 & $(.035)^{* * *}$ \\
\hline Internet use & .148 & $(.032)^{* *}$ & .069 & $(.033)^{*}$ & .114 & $(.038)^{* *}$ & .087 & $(.032)^{* *}$ & .060 & $(.035)$ & -.044 & $(.033)$ \\
\hline French-speaking & -.323 & $(.077)^{* * *}$ & .011 & (.078) & .128 & $(.080)$ & -.139 & $(.080)$ & -.139 & $(.073)$ & -.709 & $(.086)^{* * *}$ \\
\hline$\underline{\text { Italian-speaking }}$ & -.228 & (.144) & .120 & (.155) & -.007 & $(.106)$ & -.353 & $(.142)^{*}$ & -.031 & $(.140)$ & -.227 & $(.123)$ \\
\hline $\mathrm{R}^{2}$ & .101 & & .039 & & .077 & & .079 & & .017 & & .119 & \\
\hline Adj. $R^{2}$ & .094 & & .031 & & .069 & & .071 & & .008 & & .111 & \\
\hline Num. obs. & 1043 & & 1039 & & 1017 & & 1044 & & 1034 & & 1043 & \\
\hline RMSE & .945 & & .987 & & .947 & & .925 & & .980 & & .932 & \\
\hline
\end{tabular}

${ }^{* * *} \mathrm{p}<.001,{ }^{* *} \mathrm{p}<.01,{ }^{*} \mathrm{p}<.05$; standard errors in parenthesis; algorithmic-selection applications are in bold, other online services in italics. Absolute effect sizes of significant coefficients are highlighted for $>.15$ (light grey), > 3 (grey) and $>.45$ (dark grey).

is not sufficient for the assessment of its relevance and effects in daily life. In line with a comprehensive, mixed-methods measurement model of algorithmic governance (Latzer \& Festic, 2019), this paper suggests using subjectively assigned relevance as a weighting for the interpretation of other findings such as data on the amount and frequency of social media use.

Major findings according to the paper's research questions include, first, that Internet users perceive algorithmic-selection applications as less relevant in particular compared to offline but also to online alternatives. This empirically supports claims from qualitative news repertoire studies that - although increasingly used algorithmic-selection applications are unlikely to replace established sources such as traditional journalistic content for news consumption (Schmidt et al., 2019).

Second, algorithmic-selection applications, in particular social media, are found to be of relatively low assigned relevance for all life domains investigated. Offline activities are consistently ranked highest. Search engines are ranked as the most relevant algorithmic-selection applications across all life domains. This is in line with studies (Pew Research Center, 
2016; Purcell, 2011) that document the wide embeddedness of search engines in daily lives.

Third, younger and more frequent Internet users assign greater relevance to various algorithmic-selection applications across life domains. This underlines earlier findings that younger people integrate algorithmic-selection applications such as fitness trackers, music streaming, or social media more heavily in their everyday lives (Abril, 2016; Anderson, 2016; Shearer \& Matsa, 2018; Gottfried et al., 2016; Shearer, 2018; Smith, 2016). Further, people with higher educational levels are more likely to assign a lower relevance to algorithmic-selection applications than lower-educated Internet users. This result may qualify findings by the Pew Research Center (2019), that the proportion of social media users is greater for those with higher education $(79 \%)$ than for the less well educated (64\%). Subjectively assigned relevance proves beneficial as an additional dimension to weight previous findings on usage time.

Altogether, results on assigned relevance allow for a better interpretation of usage data. The relevance for people does not necessarily rise with the amount of use. Services may be highly influential, even if people report a low usage time - and vice versa. These discrepancies seem to apply in particular for social media like Facebook. Its assigned relevance is consistently very low across all life domains, including political and social orientation, where it ranks lowest. This qualifies and calls for rethink of concerns about the prevalence of risks in societies, if they are solely raised on the basis of intensive social media use.

Findings that algorithmic-selection applications are assigned a comparatively low relevance can be interpreted in two ways (Festic, 2020). On the one hand, the social relevance of algorithmic selection may generally be overestimated and - despite their high user counts and long usage times - these applications may not be so relevant after all when comparing their relevance to more traditional online and offline alternatives. On the other hand, since this article relies on self-reported assignments of relevance, it is conceiv- able that people may be misjudging the relevance of certain services and activities, algorithmic-selection applications in particular.

There could be two reasons for this: (1) there is the notion that (media) effects are often undetectable for individuals. Third-person effects may occur (Davison, 1983), people may tend to overestimate media effects on others and underestimate them on themselves (Tsay-Vogel, 2016). (2) Effects may be denied, because individuals do not want to accept the influence of algorithmic selection or because of social desirability (Holtgraves, 2004). Moreover, there may be different reasons why people under- or overestimate the relevance assigned to algorithmic-selection applications. Altogether, further research is needed to determine the likelihood of such effects on self-reported data in the respective cases.

There are a few limitations to consider when interpreting the results of this article. The selection of activities is derived from qualitative interviews conducted prior to this study and the life domains that we refer to in this study draw on a selection suggested by Büchi et al. (2016). Although meticulously aiming for saturation for these selections, neither the lists of offline and online activities nor the chosen life domains are necessarily exhaustive and simplify everyday realities. Furthermore, to allow interaction by participants, data was gathered on the subjective relevance for the specific services but not directly the actual algorithmic aspects of it. Hence, one could rate "YouTube etc." or "social media" as very relevant without being affected by its algorithmic aspects. Additionally, the degree to which the algorithmic aspects interfere with the main usage purpose of a service varies greatly.

Moreover, spillover effects between different activities and services are likely but difficult to grasp. An influencer who became famous via social media might subsequently be present on traditional TV, in print, or be the topic of offline conversations. Further investigations may resolve these relations, for example, by explicitly asking for such instances. Taking all lim- 
itations into account, it is likely that people underestimate the actual relevance of algorithmic-selection applications, "YouTube etc." and "social media" especially, for their lives.

Finally, cultural differences between countries should be taken into consideration. Our representative results on the relevance assigned by the Swiss population should not be applied uncritically to qualify empirical usage data from countries with a quite different cultural imprint.

\section{Conclusion}

An adequate and up-to-date understanding of the social relevance of algorithmic selection is a prerequisite when aiming to regulate algorithmic selection. Applications that are based on algorithmic selection have been under public scrutiny for bearing a plethora of risks. For example, algorithmically curated social media feeds are claimed to be responsible for manipulation and the distribution of biased information. From a public-policy perspective, this raises questions about the need for regulatory measures. Choosing an appropriate governance of algorithmic-selection applications can be supported by accurate knowledge about their social relevance. This includes, for example, information on how strongly Internet users actually rely on social media for their daily social and political orientation, what other sources they consult and how much they know about the process of algorithmic selection. Only such a thorough empirical investigation can form an appropriate basis for assessing the magnitude of risks that might be induced by algorithmic selection and consequentially the adequate governance measures. This paper adds to the debate on the relevance of algorithmic selection in two ways.

First, it introduces subjectively assigned relevance as an additional approach to weight findings on the overall social relevance of algorithmic selection that rely on measures of the frequency and amount of use of algorithmic-selection applications. The combination of these measures can lead to a more realistic assessment of the matter at hand, allowing more appropriate policy decisions.

Second, by taking a user perspective and analyzing subjectively assigned relevance on a nation-wide, representative level for five different life domains, the paper directly adds to a more comprehensive and nuanced empirical understanding of the social relevance of algorithmic selection, provides novel empirical insights for the ongoing debate and informs policy-makers aiming for adequate governance decisions.

According to the findings, young as well as heavy Internet users assigned a high relevance to algorithmic-selection applications. As a result, members of these groups are more likely to be exposed to risks associated with algorithmic selection. To mitigate these risks, policymakers should focus on these high-risk groups when attempting to raise awareness regarding potentially negative consequences of algorithmic selection.

In addition to existing self-reported data on the amount and frequency of use and this paper's approach to subjectively assigned relevance, further research could include tracking data, for example, to reduce problems with inaccurate and biased self-reporting. This would facilitate an improved assessment of the social relevance of algorithmic-selection applications.

With slight variations across life domains and socio-demographic groups, this article suggests that Internet users generally perceive algorithmic-selection applications as not overwhelmingly relevant for their everyday lives. Within this group of services, search engines are assigned a relatively high and social media a very low relevance. Although potential risks should not be trivialized, these findings render the image of an Internet user who is at the mercy of algorithms and strategies of platform companies slightly less likely. Differences identified in the relevance assigned to algorithmic-selection applications in societies should be appropriately considered in the assessment and governance of chances and risks they pose for them. 


\section{Acknowledgement}

This research project received funding from the Swiss National Science Foundation (SNF Grant No. 176443).

\section{Supplementary material}

Supplementary material for this article is available online in the format provided by the authors (unedited). https:// www.hope.uzh.ch/scoms/article/view/j. scoms.2021.01.005

\section{References}

Abril, E. P. (2016). Tracking myself: Assessing the contribution of mobile technologies for self-trackers of weight, diet, or exercise. Journal of Health Communication, 21(6), 638-646. https://doi.org/10.1080/1081073 0.2016 .1153756

Adapa, A., Nah, F. F.-H., Hall, R. H., Siau, K., \& Smith, S. N. (2018). Factors influencing the adoption of smart wearable devices. International Journal of Human-Computer Interaction, 34(5), 399-409. https://doi.org /10.1080/10447318.2017.1357902

Althaus, S. L., \& Tewksbury, D. (2000). Patterns of Internet and traditional news media use in a networked community. Political Communication, 17(1), 21-45. https://doi. org/10.1080/105846000198495

Anderson, M. (2016). More Americans using smartphones for getting directions, streaming TV. Retrieved from https://www. pewresearch.org/fact-tank/2016/01/29/ us-smartphone-use/.

Araujo, T., Wonneberger, A., Neijens, P., \& de Vreese, C. (2017). How much time do you spend online? Understanding and improving the accuracy of self-reported measures of Internet use. Communication Methods and Measures, 11(3), 173-190. https://doi. org/10.1080/19312458.2017.1317337

Baek, K., \& Kim, Y. (2016). Exploring the relationship between mobile application use and political information seeking and political discussion. Journal of Information Technology \& Politics, 13(2), 175-186. https://doi.org/10.1080/19331681.2016.1 160264

Bayer, J., Bitiukova, N., Bard, P., Szakács, J., Alemanno, A., \& Uszkiewicz, E. (2019). Disinformation and Propaganda - Impact on the Functioning of the Rule of Law in the EU and its Member States. HEC Paris Research Paper No. LAW-2019-1341. https:// doi.org/10.2139/ssrn.3409279

Beer, D. (2017). The social power of algorithms. Information, Communication \& Society, 20(1), 1-13. https://doi.org/10.1080/13691 18X.2016.1216147

Bialik, K., \& Matsa, K. E. (2017). Key trends in social and digital news media. Retrieved from https://www.pewresearch.org/facttank/2017/10/04/key-trends-in-socialand-digital-news-media/

Bucher, T. (2012). Want to be on the top? Algorithmic power and the threat of invisibility on Facebook. New Media \& Society, 14(7), 1164-1180. https://doi. org/10.1177/1461444812440159

Bucher, T. (2017). The algorithmic imaginary: Exploring the ordinary affects of Facebook algorithms. Information, Communication \& Society, 20(1), 30-44. https://doi.org /10.1080/1369118X.2016.1154086

Büchi, M., Just, N., \& Latzer, M. (2016). Modeling the second-level digital divide: A five-country study of social differences in Internet use. New Media \& Society, 18(11), 2703-2722. https://doi. org/10.1177/1461444815604154

Canhoto, A. I., \& Arp, S. (2017). Exploring the factors that support adoption and sustained use of health and fitness wearables. Journal of Marketing Management, 33(12), 32-60. https://doi.org/10.1080/026725 7X.2016.1234505

Caplan, R., \& Boyd, D. (2018). Isomorphism through algorithms: Institutional dependencies in the case of Facebook. Big Data \& Society, 5(1), 1-12. https://doi. org/10.1177/2053951718757253

Celik, M., \& Dokuz, A. S. (2018). Discovering socially similar users in social media datasets based on their socially important locations. Information Processing \& Management, 54(6), 1154-1168. https://doi. org/10.1016/j.ipm.2018.08.004

Chen, H. (2012). The impact of comments and recommendation system on online shop- 
per buying behaviour. Journal of Networks, 7(2), 345-350.

Courtois, C., \& Timmermans, E. (2018). Cracking the Tinder Code: An Experience Sampling Approach to the Dynamics and Impact of Platform Governing Algorithms. Journal of Computer-Mediated Communication, 23(1), 1-16. https://doi. org $/ 10.1093 / \mathrm{jcmc} / \mathrm{zmx} 001$

Davison, W. P. (1983). The third-person effect in communication. Public Opinion Quarterly, 47(1), 1-15. https://doi. org $/ 10.1086 / 268763$

De Keyzer, F., Dens, N., \& De Pelsmacker, P. (2015). Is this for me? How Consumers Respond to Personalized Advertising on Social Network Sites. Journal of Interactive Advertising, 15(2), 124-134. https://doi.org /10.1080/15252019.2015.1082450

de Vreese, C. H. \& Neijens, P., (2016). Measuring media exposure in a changing communications environment. Communication Methods and Measures, 10(2-3), 69-80. https://doi.org/10.1080/19312458.2016.1 150441

Deng, T., Kanthawala, S., Meng, J., Peng, W., Kononova, A., Hao, Q., Zhang, Q., \& David, P. (2019). Measuring smartphone usage and task switching with log tracking and self-reports. Mobile Media \& Communication, 7(1), 3-23. https://doi. org/10.1177/2050157918761491

Diakopoulos, N. (2015). Algorithmic accountability: Journalistic investigation of computational power structures. Digital Journalism, 3(3), 398-415. https://doi.org/10.1 080/21670811.2014.976411

Dimmick, J., Chen, Y., \& Li, Z. (2004). Competition between the Internet and traditional news media: The gratification-opportunities niche dimension. Journal of Media Economics, 17(1), 19-33. https://doi. org/10.1207/s15327736me1701_2

eMarketer. (2020). Western europe ecommerce trends 2020. Consumer-facing, AI, social commerce and delivery/returns on the agenda. Retrieved from https://www. emarketer.com/content/western-europe-ecommerce-trends- 2020.

European Commission. (2018). Social media and networks, innovation and policy. Shaping Europe's Digital Future - European Commission. Retrieved from https:// ec.europa.eu/digital-single-market/en/ social-media-and-networks-innovation-and-policy.

Festic, N. (2020). Same, same but different! Qualitative evidence on how algorithmic selection applications govern different life domains. Regulation \& Governance. Advance online publication. https://doi. org/10.1111/rego.12333

Gil de Zúñiga, H., Ardèvol-Abreu, A., \& Casero-Ripollés, A. (2021). WhatsApp political discussion, conventional participation and activism: Exploring direct, indirect and generational effects. Information, Communication \& Society, 24(2), 201-218. https:// doi.org/10.1080/1369118X.2019.1642933

Gillespie, T. (2014). The relevance of algorithms. In T. Gillespie, P. J. Boczkowski, \& K. A. Foot (Eds.), Media Technologies (pp. 167-194). Cambridge, Mass.: The MIT Press. https://doi.org/10.7551/mitpress/9780262525374.003.0009

Gottfried, J., Barthel, M., Shearer, E., \& Mitchell, A. (2016). The 2016 presidential campaign - a news event that's hard to miss. Retrieved from https://www.journalism. org/2016/02/04/the-2016-presidentialcampaign-a-news-event-thats-hard-tomiss/

Guess, A., Munger, K., Nagler, J., \& Tucker, J. (2019). How accurate are survey responses on social media and politics? Political Communication, 36(2), 241-258. https:// doi.org/10.1080/10584609.2018.1504840

Hamilton, C. (2019). Popular music, digital technologies and data analysis: New methods and questions. Convergence: The International Journal of Research into New Media Technologies, 25(2), 225-240. https:// doi.org/10.1177/1354856519831127

Hasebrink, U., \& Domeyer, H. (2012). Media repertoires as patterns of behaviour and as meaningful practices: A multimethod approach to media use in converging media environments. Journal of Audience \& Reception Studies, 9(2), 757-779.

Hasebrink, U., \& Hepp, A. (2017). How to research cross-media practices? Investigating media repertoires and media ensembles. Convergence: The International Journal of Research into New Media Technologies, 23(4), 362-377. https://doi. org/10.1177/1354856517700384 
Holtgraves, T. (2004). Social desirability and self-reports: Testing models of socially desirable responding. Personality and Social Psychology Bulletin, 30(2), 161-172. https://doi. org/10.1177/0146167203259930

Junco, R. (2013). Comparing actual and self-reported measures of Facebook use. Computers in Human Behavior, 29(3), 626-631. https://doi.org/10.1016/j. chb.2012.11.007

Jürgens, P., Stark, B., \& Magin, M. (2019). Two half-truths make a whole? On bias in self-reports and tracking data. Social Science Computer Review, 38(5), 600-615. https://doi. org/10.1177/0894439319831643

Just, N., \& Latzer, M. (2017). Governance by algorithms: Reality construction by algorithmic selection on the Internet. Media, Culture \& Society, 39(2), 238-258. https:// doi.org/10.1177/0163443716643157

Karakaya, S., \& Glazier, R. A. (2019). Media, information, and political participation: The importance of online news sources in the absence of a free press. Journal of Information Technology \& Politics, 16(3), 290-306. https://doi.org/10.1080/1933168 1.2019.1645784

Kilger, M., \& Romer, E. (2013). Using passive measurement to describe reading app behaviors in a rich media mobile environment. Paper presented at the Print and Digital Research Forum, Nice.

Kim, H., \& Huh, J. (2017). Perceived relevance and privacy concern regarding online behavioral advertising (OBA) and their role in consumer responses. Journal of Current Issues \& Research in Advertising, 38(1), 92-105. https://doi.org/10.1080/10641734 .2016 .1233157

Kitchin, R. (2017). Thinking critically about and researching algorithms. Information, Communication \& Society, 20(1), 14-29. https://doi.org/10.1080/136911 8X.2016.1154087

Latzer, M., Büchi, M., \& Festic, N. (2020). Internet Use in Switzerland 2011-2019: Trends, Attitudes and Effects. Summary Report from the World Internet Project - Switzerland. University of Zürich. Retrieved from https://mediachange.ch/media//
pdf/publications/SummaryReport_WIPCH_2019.pdf.

Latzer, M., \& Festic, N. (2019). A guideline for understanding and measuring algorithmic governance in everyday life. Internet Policy Review, 8(2), 1-19. https://doi. org/10.14763/2019.2.1415

Latzer, M., Hollnbuchner, K., Just, N., \& Saurwein, F. (2016). The economics of algorithmic selection on the Internet. In J. Bauer \& M. Latzer (Eds.), Handbook on the Economics of the Internet (pp. 395-425). Cheltenham, UK: Edward Elgar. https:/ / doi.org/10.4337/9780857939852

Latzer, M., \& Just, N. (2020). Governance by and of algorithms on the Internet: Impact and consequences. In Oxford Research Encyclopedia of Communication. Oxford, UK: Oxford University Press. https://doi.org/10.1093/acrefore/9780190228613.013.904

Lee, F. L. F., Lee, P. S. N., So, C. Y., Leung, L., \& Chan, M. C. (2017). Conditional impact of facebook as an information source on political opinions: The case of political reform in Hong Kong. Asian Journal of Political Science, 25(3), 365-382. https://doi. org/10.1080/02185377.2017.1352523

Lepa, S., \& Hoklas, A.-K. (2015). How do people really listen to music today? Conventionalities and major turnovers in German audio repertoires. Information, Communication \& Society, 18(10), 1253-1268. https://doi.or g/10.1080/1369118X.2015.1037327

Lin, Z. (2014). An empirical investigation of user and system recommendations in e-commerce. Decision Support Systems, 68, 111-124. https://doi.org/10.1016/j. dss.2014.10.003

Logg, J. M., Minson, J. A., \& Moore, D. A. (2019). Algorithm appreciation: People prefer algorithmic to human judgment. Organizational Behavior and Human Decision Processes, 151, 90-103. https://doi. org/10.1016/j.obhdp.2018.12.005

Lomborg, S., \& Frandsen, K. (2016). Self-tracking as communication. Information, Communication \& Society, 19(7), 10151027. https://doi.org/10.1080/136911 8X.2015.1067710

Mattlin, J. A., \& Gagen, J. (2013). Passive measurement of print revisited: The case of 
digital magazines. Paper presented at the Print and Digital Research Forum, Nice.

Möller, J., Trilling, D., Helberger, N., \& Es, B. van. (2018). Do not blame it on the algorithm: An empirical assessment of multiple recommender systems and their impact on content diversity. Information, Communication \& Society, 21(7), 959-977. https://doi.org/10.1080/136911 8x.2018.1444076

Newman, N., Fletcher, R., Schulz, A., Andı, S., \& Nielsen, R. (2020). Reuters institute digital news report 2020. Reuters Institute for the Study of Journalism. Retrieved from https://reutersinstitute.politics.ox.ac.uk/ sites/default/files/2020-06/DNR_2020_FINAL.pdf.

Pariser, E. (2011). The filter bubble: What the Internet is hiding from you. New York, NY: Penguin Press.

Park, C. S. (2019). Learning politics from social media: Interconnection of social media use for political news and political issue and process knowledge. Communication Studies, 70(3), 253-276. https://doi.org/10. 1080/10510974.2019.1581627

Pew Research Center. (2016). Searching for news: The flint water crisis. Retrieved from https://www.journalism.org/essay/ searching-for-news/

Pew Research Center. (2019). Social media fact sheet. Retrieved from https://www.pewinternet.org/fact-sheet/social-media/

Phelan, C., Lampe, C., \& Resnick, P. (2016). It's Creepy, But it Doesn't Bother Me. Proceedings of the 2016 CHI Conference on Human Factors in Computing Systems - CHI '16, 5240-5251. https://doi. org/10.1145/2858036.2858381

Picone, I., Courtois, C., \& Paulussen, S. (2015). When news is everywhere: Understanding participation, cross-mediality and mobility in journalism from a radical user perspective. Journalism Practice, 9(1), 35-49. https://doi.org/10.1080/17512786.2014.9 28464

Promberger, M., \& Baron, J. (2006). Do patients trust computers? Journal of Behavioral Decision Making, 19(5), 455-468. https:// doi.org/10.1002/bdm.542

Purcell, K. (2011). Search and email still top the list of most popular online activities. Retrieved from https://www.pewinternet. org/2011/08/09/search-and-email-stilltop-the-list-of-most-popular-online-activities/

Quan-Haase, A., \& Young, A. L. (2010). Uses and gratifications of social media: A comparison of Facebook and instant messaging. Bulletin of Science, Technology \& Society, 30(5), 350-361. https://doi. org/10.1177/0270467610380009

Rains, S. A. (2007). Perceptions of traditional information sources and use of the world wide web to seek health information: Findings from the health information national trends survey. Journal of Health Communication, 12(7), 667-680. https:// doi.org/10.1080/10810730701619992

Rosenberg, S. (2008). Dreaming in code: Two dozen programmers, three years, 4,732 bugs, and one quest for transcendent software (1st pbk. ed). New York, NY: Three Rivers Press.

Scharkow, M. (2016). The accuracy of self-reported Internet use - A validation study using client log data. Communication Methods and Measures, 10(1), 13-27. https://doi.org/10.1080/19312458.2015.1 118446

Schmidt, J.-H., Merten, L., Hasebrink, U., Petrich, I., \& Rolfs, A. (2019). How do intermediaries shape news-related media repertoires and practices? Findings from a qualitative study. International Journal of Communication, 13, 853-873. Retrieved from https://ijoc.org/index.php/ijoc/article/view/9080/2572

Seaver, N. (2019). Captivating algorithms: Recommender systems as traps. Journal of Material Culture, 24(4), 421-436. https:// doi.org/10.1177/1359183518820366

Shearer, E. (2018). Social media outpaces print newspapers in the U.S. as a news source. Retrieved from https://www.pewresearch. org/fact-tank/2018/12/10/social-mediaoutpaces-print-newspapers-in-the-u-s-asa-news-source/

Shearer, E., Matsa, K. E. (2018). News use across social media platforms 2018. Retrieved from https://www.journalism. org/2018/09/10/news-use-across-socialmedia-platforms-2018/.

Smit, E. G., Van Noort, G., \& Voorveld, H. A. M. (2014). Understanding online behavioural advertising: User knowledge, privacy 
concerns and online coping behaviour in Europe. Computers in Human Behavior, 32, 15-22. https://doi.org/10.1016/j. chb.2013.11.008

Smith, A. (2016). 15\% of American adults have used online dating sites or mobile dating apps. Retrieved from https://www. pewinternet.org/2016/02/11/15-percentof-american-adults-have-used-onlinedating-sites-or-mobile-dating-apps/

Sumter, S. R., \& Vandenbosch, L. (2019). Dating gone mobile: Demographic and personality-based correlates of using smartphone-based dating applications among emerging adults. New Media \& Society, 21(3), 655-673. https://doi. org/10.1177/1461444818804773

Swart, J., Peters, C., \& Broersma, M. (2017). Navigating cross-media news use: Media repertoires and the value of news in everyday life. Journalism Studies, 18(11), 1343-1362. https://doi.org/10.1080/14616 70X.2015.1129285

Thorson, K., Cotter, K., Medeiros, M., \& Pak, C. (2021). Algorithmic inference, political interest, and exposure to news and politics on Facebook. Information, Communication \& Society, 24(2), 183-200. https://doi. org/10.1080/1369118X.2019.1642934

Tsay-Vogel, M. (2016). Me versus them: Third-person effects among Facebook users. New Media \& Society, 18(9), 1956-1972. https://doi. org/10.1177/1461444815573476

van der Nagel, E. (2018). "Networks that work too wel": Intervening in algorithmic connections. Media International Australia, 168(1), 81-92. https://doi.org/10.1177/1329878X18783002

Vraga, E. K., \& Tully, M. (2021). News literacy, social media behaviors, and skepticism toward information on social media. Information, Communication \& Society, 24(2), 150-166. https://doi.org/10.1080/136911 8X.2019.1637445

Wang, S. (2020). Calculating dating goals: Data gaming and algorithmic sociality on Blued, a Chinese gay dating app. Information, Communication \& Society, 23(2), 181-197. https://doi.org/10.1080/136911 8X.2018.1490796

Westerwick, A., Johnson, B. K., \& KnoblochWesterwick, S. (2017). Confirmation biases in selective exposure to political online information: Source bias vs. content bias. Communication Monographs, 84(3), 343-364. https://doi.org/10.1080/0363775 1.2016 .1272761

Whiting, A., \& Williams, D. (2013). Why people use social media: A uses and gratifications approach. Qualitative Market Research: An International Journal, 16(4), 362-369. https://doi.org/10.1108/QMR-06-20130041

Williamson, B. (2015). Algorithmic skin: Healthtracking technologies, personal analytics and the biopedagogies of digitized health and physical education. Sport, Education and Society, 20(1), 133-151. https://doi.org /10.1080/13573322.2014.962494

Willson, M. (2017). Algorithms (and the) everyday. Information, Communication \& Society, 20(1), 137-150. https://doi.org/10.10 80/1369118X.2016.1200645

Yang, A., \& Men, L. R. (2020). Political information use on WeChat and political discussion in China: Toward a networked political discussion model. Chinese Journal of Communication, 13(2), 129-147. https:// doi.org/10.1080/17544750.2019.1618354 